\title{
Morphological and Physiological Characteristics of Shading Tolerant and Sensitive Mungbean Genotypes
}

\author{
TITIK SUNDARI
}

\author{
Researcher of the Indonesian Legume and Tuber Crops Research Institute \\ Jalan Raya Kendalpayak Km. 8, Pakisaji-Malang, Kotak Pos 66, Malang 65101 \\ Phone: +62-341-801468, Fax: +62-341-801496, E-mail: titik_iletri@yahoo.com
}

Received October 9, 2008/Accepted December 2, 2009

\begin{abstract}
Study of morphological and physiological characteristics of the tolerant and sensitive mungbean genotypes to shading was carried out in the Station Research of the Indonesian Legume and Tuber Crops Research Institute (ILETRI) from September to December 2004. Nine tolerant genotypes (MMC 87 D-KP-2, MLG 369, MLG 310, MLG 424, MLG 336, MLG 428, MLG 237, MLG 429, and VC2768B) and three sensitive genotypes to shading (Nuri, MLG 460, and MLG 330) were tested in two shading levels, that were without shading and shading of $52 \%$. The randomized complete block design with three replications analysis. The results showed that leaf characters of shading tolerant and sensitive genotypes were different. The shading tolerant mungbean genotypes had good response to light stress so that the growth and development of the leaves were better than that of sensitive genotypes. The shading tolerant mungbean genotypes had bigger and thicker leaves than that of sensitive genotypes. The shading treatments caused reducing rate of PAR absorption, transpiration, photosynthesis, and $\mathrm{CO}_{2}$ stomata conductance. The reduction of all parameters in tolerant genotype was smaller than that of sensitive genotype. The specific leaf area at four weeks after planting could be used as shading tolerant indicator of mungbeans.
\end{abstract}

Key words: mungbean, characteristics, morphology, physiology, leaves, tolerant, sensitive, shading

\section{INTRODUCTION}

Shading is a factor affecting the plant productivity at plantations of agriculture as well as forestry crop. Shading was an important phenomenon to be known and if it was possibly to be controlled. Shading will cause decreasing of quantity and quality of the sunlight intercept to the crop, and it will affect the productivity of photosynthesis.

Respones of most crop to change in light intensity varies depend on the species (Thompson et al. 1992). Light intensity requirement of each plant species was different depended on the age, environment condition, and length of day. The physiological behavior of young plants of A. rosaeodora to different light intensities suggests that its photosynthetic apparatus was more efficient when they were grown on medium light intensity $\left(500 \mathrm{a} 1000 \mu \mathrm{mol} \cdot \mathrm{m}^{-2} \cdot \mathrm{s}^{-1}\right)$. However, their photosynthetic activity were suppessed when they were grown in low or height light intensity (de Carvalhu Gonsales et al. 2005).

Effect of low irradiance on leaf of $\mathrm{C}_{3}$ plants lead to phenotypic changes in their photosynthetic apparatus. The shade tolerant species had wider leaves, and played a role to the absorbtion of photosynthetically active radiation (PAR) under low irradiance (St-Jacques et al. 1991; Poorter et al. 1995). The aim of this research was to determine leaf morphological and physiological characteristics of mungbean shading tolerant and sensitive genotypes.

\section{MATERIALS AND METHODS}

The research was conducted in the Kendalpayak research station of the Indonesian Legume and Tuber Crops Research Institute (ILETRI) from September to December 2004. The place was located at $450 \mathrm{~m}$ above sea level, which had Entisol soil and with $\mathrm{C} 3$ climate type according to Oldeman. Nine shading tolerant genotypes of mungbean (MMC 87 D-KP-2, MLG 369, MLG 310, MLG 424, MLG 336, MLG 428, MLG 237, MLG 429, and VC2768B) and three sensitive genotypes (Nuri, MLG 460 and MLG 330) were tested on two different shading levels, i.e. without shading and $52 \%$ shading. The experiment was set up using randomized complete block design with three replications.

The treatment of 52\% shading was set up using two layers of black screen at the high of $1.8 \mathrm{~m}$ from soil surface. The light intensity was measured using Lux meter.

Each genotype was planted five seeds per hole, with planting distance of $0.40 \times 0.15 \mathrm{~m}$. Fertilization was applied at the time of planting with $50 \mathrm{~kg} \mathrm{ha}^{1} \mathrm{Urea}, 50 \mathrm{~kg} \mathrm{ha}^{1} \mathrm{KCl}$, and $100 \mathrm{~kg} \mathrm{ha}{ }^{1}$ SP36. The thinning was conducted at ten days after planting by leaving two crops per hole. The thinning and weeding were done at four weeks after planting (WAP). The pest and disease control, were conducted regularly every three days.

Observation of leaf number, leaf area, specific leaf area (SLA), rate of PAR absorbtion, photosynthesis, transpiration 
activities, the stomatal $\mathrm{CO}_{2}$ conductance and chlorophyll content was conducted regularly every two weeks from four weeks up to harvest. The leaf area was measured by the gravimetric methods (Sitompul \& Guritno 1991). The specific leaf area (SLA) was measured by the SLA $=((\mathrm{LAi} / \mathrm{LDWi})$. The chlorophyll content was observed using spectrophotometric method. The rate of PAR absorption, photosynthesis, transpiration and stomatal $\mathrm{CO}_{2}$ conductance were observed using LCi Portable Photosynthesis System (3015). The data were statistically analyzed using Duncan's test in the 5\% á level and Contrast of Ortogonal test.

\section{RESULTS}

Effect of Shading on Leaf Number and Morphology. Leaf number at six and eight WAP was difference (Table 1). Generally, leaf number at without shading treatment more than that of shading treatment. The greatest leaf number at six WAP was achieved by MLG 429 genotype and the fewest was found on MLG 460 genotype. The greatest leaf number at eight WAP was achieved by Nuri genotype and the fewest was found on MLG 424, MLG 460, and MLG 330 genotypes. Interaction of genotype and shading also had a significant effect to leaf area (Figure 1). Except MLG 424 genotype, all tested genotypes showed negative response to shading.

In four and eight WAP, interaction between genotype and shading showed the significant effect on the specific leaf area (SLA) (Table 1). SLA was increased sharply at the initial growth, then it was constant at initial generative phase and it was decline in the further phases (Table 1).
Results of the contrast test (Table 2) showed that leaf area and morphology of shading tolerant genotypes were different from shading sensitive genotypes. Leaf area of shading tolerant genotype was bigger than that of sensitive genotype at four and six WAP. Leaf number of shading tolerant and sensitive genotypes did not show the difference, however leaf number reducing of tolerant genotype was relatively less than that of sensitive genotype, especially at six and eight WAP. SLA values of shading tolerant and sensitive genotypes did not show significant difference, except at four WAP. At four WAP, SLA value of shading tolerant genotypes smaller than that of sensitive genotypes. It mean that leaves of shading tolerant genotypes were thicker than that of sensitive genotypes (Table 2).

Plant Physiology. Photosynthetically active radiation (PAR) absorption rate of each genotype showed significant difference (Figure 2). The result showed that shading treatment caused reduction of PAR absorption rate at four and six WAP. The highest reduction of PAR absorption rate was reached by MLG 429 genotype and the lowest was found on MLG 424 and MLG 428 genotypes at four and six WAP respectively. At eight WAP, the highest reduction of PAR absorption rate was reached by MLG 310 genotype and the lowest by VC 2768B genotype (Figure 2).

Genotype and shading interaction had significant effect to transpiration rate (Figure 3 ). The shading treatment could reduce transpiration rate. At four WAP, the highest reduction of transpiration rate was achieved by MLG 429 genotype and the lowest was found on MLG 369 genotype (Figure 3a). The highest reducing of transpiration rate in six WAP was reached

Table 1. Leaf number per plant and specific leaf area $\left(\mathrm{cm}^{2} / \mathrm{g}\right)$ of twelve mungbean genotypes at two shading levels

\begin{tabular}{|c|c|c|c|c|c|c|c|}
\hline \multirow{2}{*}{ Shade $(\%)$} & \multirow{2}{*}{ Genotypes } & \multicolumn{3}{|c|}{ Leaf number at: } & \multicolumn{3}{|c|}{ Specific leaf area $\left(\mathrm{cm}^{2} / \mathrm{g}\right)$ at: } \\
\hline & & 4 WAP & $6 \mathrm{WAP}$ & $8 \mathrm{WAP}$ & 4 WAP & 6 WAP & 8 WAP \\
\hline 0 & MMC 87 D-KP-2 & 12 & $22 \mathrm{efgh}$ & $21 \mathrm{e}$ & $276.8 \mathrm{efg}$ & 214.8 & $140.6 \mathrm{k}$ \\
\hline 0 & MLG 369 & 13 & $26 \mathrm{de}$ & $26 \mathrm{c}$ & $267.1 \mathrm{efg}$ & 220.2 & $189.8 \mathrm{gh}$ \\
\hline 0 & MLG 310 & 13 & $25 \mathrm{ef}$ & $26 c$ & $285.5 \mathrm{ef}$ & 216.8 & $192.2 \mathrm{~g}$ \\
\hline 0 & MLG 424 & 13 & $22 \mathrm{efg}$ & $20 \mathrm{ef}$ & $347.9 d$ & 218.8 & 175.2ghi \\
\hline 0 & MLG 336 & 14 & $24 \mathrm{ef}$ & $29 \mathrm{~b}$ & $240.4 \mathrm{hi}$ & 216.8 & $160.4 \mathrm{ijk}$ \\
\hline 0 & VC $2768 \mathrm{~B}$ & 14 & 26de & $24 d$ & $286.8 \mathrm{e}$ & 190.0 & $148.6 \mathrm{jk}$ \\
\hline 0 & MLG 428 & 14 & $18 \mathrm{ghij}$ & $27 \mathrm{c}$ & $284.0 \mathrm{ef}$ & 199.1 & $165.7 \mathrm{ij}$ \\
\hline 0 & MLG 237 & 13 & $29 \mathrm{~cd}$ & $26 \mathrm{c}$ & $236.8 \mathrm{i}$ & 194.4 & $162.6 \mathrm{ijk}$ \\
\hline 0 & MLG 429 & 15 & $41 \mathrm{a}$ & $26 \mathrm{c}$ & $257.9 \mathrm{ghi}$ & 232.2 & $235.2 \mathrm{def}$ \\
\hline 0 & Nuri* & 16 & $36 \mathrm{~b}$ & $32 \mathrm{a}$ & $288.1 \mathrm{e}$ & 226.2 & $179.4 \mathrm{ghi}$ \\
\hline 0 & MLG $460 *$ & 15 & $22 \mathrm{efg}$ & $18 \mathrm{fg}$ & $281.2 \mathrm{efg}$ & 219.7 & $193.1 \mathrm{~g}$ \\
\hline 0 & MLG 330* & 13 & $31 c^{\circ}$ & $26 \mathrm{c}$ & $261.4 \mathrm{fgh}$ & 211.5 & $168.3 \mathrm{hij}$ \\
\hline 52 & MMC 87 D-KP-2 & 11 & $17 \mathrm{ij}$ & 16hi & $338.3 \mathrm{~d}$ & 252.3 & $193.0 \mathrm{~g}$ \\
\hline 52 & MLG 369 & 12 & $16 \mathrm{ij}$ & $15 \mathrm{i}$ & $376.1 \mathrm{bc}$ & 310.9 & $268.1 \mathrm{abc}$ \\
\hline 52 & MLG 310 & 11 & $16 \mathrm{ij}$ & $15 \mathrm{i}$ & $371.1 \mathrm{c}$ & 304.1 & $246.3 \mathrm{cde}$ \\
\hline 52 & MLG 424 & 13 & 19ghij & $14 \mathrm{i}$ & $342.2 \mathrm{~d}$ & 314.4 & $255.6 \mathrm{bcd}$ \\
\hline 52 & MLG 336 & 13 & $17 \mathrm{ij}$ & $15 \mathrm{i}$ & $383.2 \mathrm{bc}$ & 287.8 & $250.2 \mathrm{~cd}$ \\
\hline 52 & VC $2768 B$ & 12 & $17 \mathrm{hij}$ & $17 \mathrm{gh}$ & $399.6 \mathrm{ab}$ & 294.9 & $226.2 \mathrm{ef}$ \\
\hline 52 & MLG 428 & 12 & 19ghij & $18 \mathrm{~g}$ & $379.7 \mathrm{bc}$ & 285.3 & $218.7 \mathrm{f}$ \\
\hline 52 & MLG 237 & 15 & $22 \mathrm{efgh}$ & $20 \mathrm{e}$ & $344.9 \mathrm{~d}$ & 300.6 & $277.6 \mathrm{ab}$ \\
\hline 52 & MLG 429 & 14 & $23 \mathrm{efg}$ & $21 \mathrm{e}$ & $396.7 \mathrm{ab}$ & 344.9 & $289.2 \mathrm{a}$ \\
\hline 52 & Nuri* & 13 & $21 \mathrm{fghi}$ & $21 \mathrm{e}$ & $417.9 \mathrm{a}$ & 322.8 & $276.0 \mathrm{ab}$ \\
\hline 52 & MLG $460^{*}$ & 14 & $15 \mathrm{j}$ & $14 \mathrm{i}$ & $389.6 \mathrm{bc}$ & 253.1 & $197.1 \mathrm{~g}$ \\
\hline 52 & MLG $330^{*}$ & 12 & 19ghij & $14 \mathrm{i}$ & $382.6 b c$ & 323.2 & $244.6 \mathrm{cde}$ \\
\hline \multicolumn{2}{|c|}{ Variance coefficient $(\%)$} & 11.03 & 11.03 & 10.42 & 4.07 & 12.85 & 6.21 \\
\hline
\end{tabular}



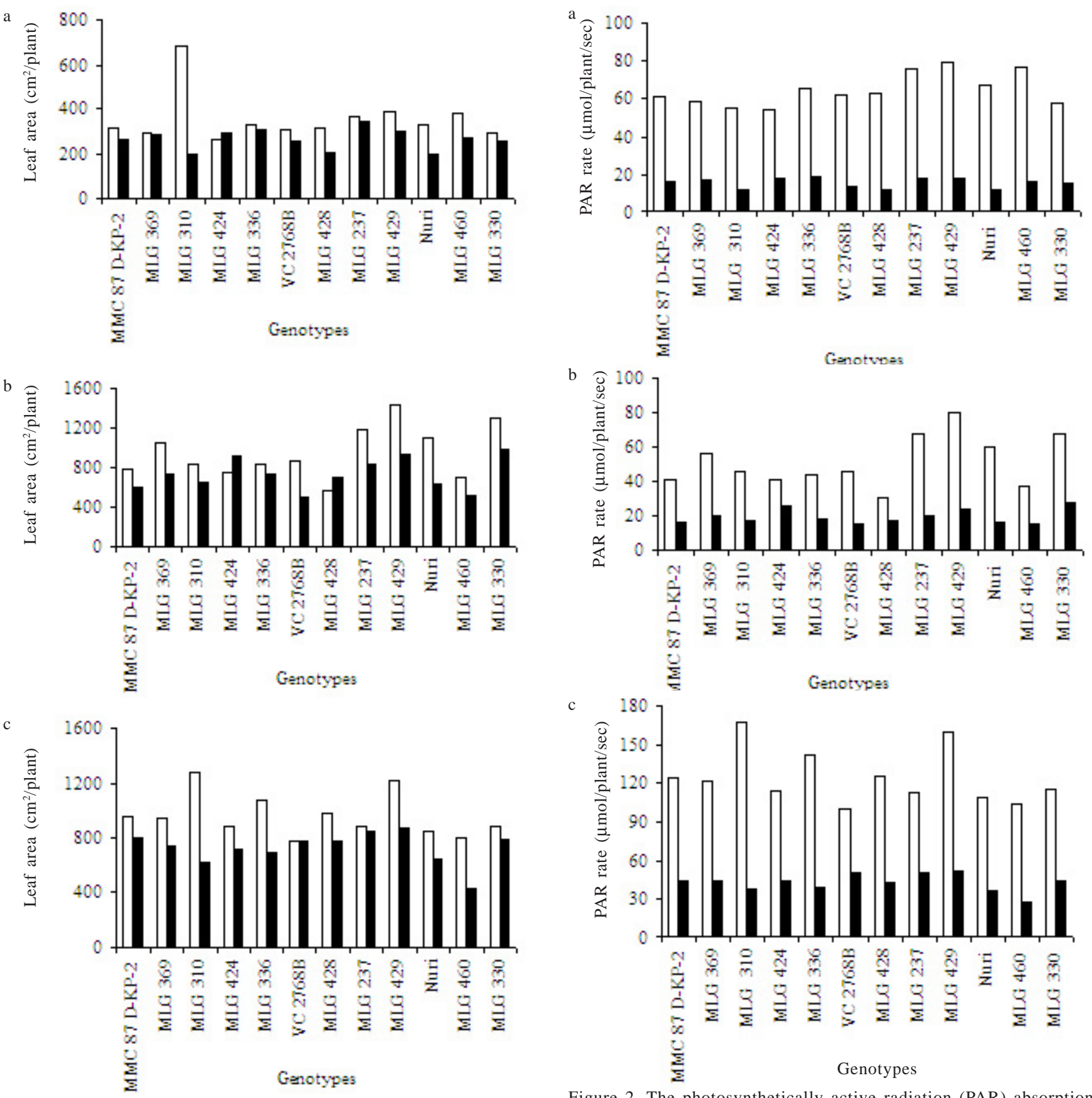

Figure 1. The leaf area ( $\mathrm{cm}^{2} /$ plant) at: (a) 4, (b) 6, and (c) 8 WAP of twelve mungbean genotypes in without shading and 52\% shading level. $\square$ : without shading, $\square: 52 \%$ shading.

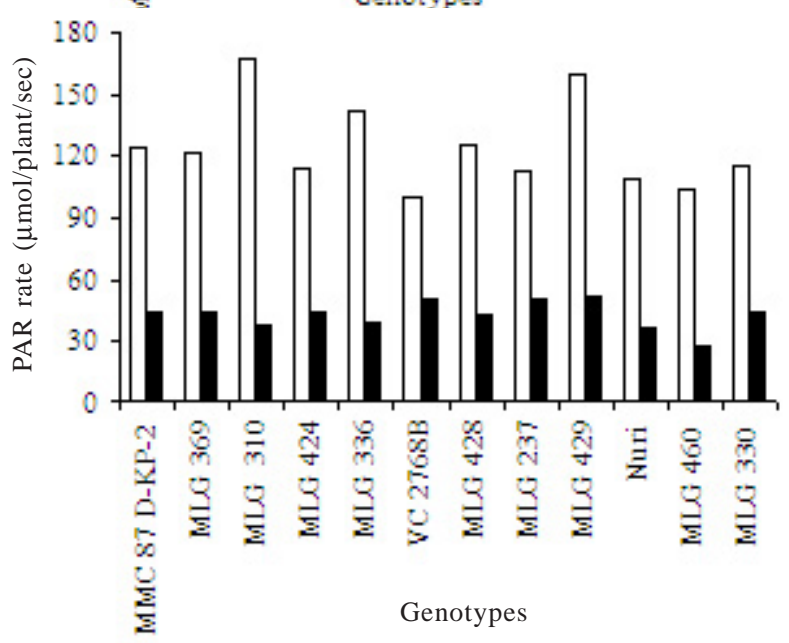

Figure 2. The photosynthetically active radiation (PAR) absorption rate (ì mol/plant/sec) at: (a) 4, (b) 6 , and (c) 8 WAP of twelve mungbean genotypes in without shading and 52\% shading level. $\square$ : without shading, $\mathbf{\square}$ : $52 \%$ shading.

Table 2. The contrast test of the leaf character inter-group of mungbean genotypes in the 0 and $52 \%$ shading level

\begin{tabular}{|c|c|c|c|c|c|c|}
\hline \multirow{3}{*}{ Quantitative characters } & \multicolumn{4}{|c|}{ Shading levels } & \multicolumn{2}{|c|}{ Percentage change $(\%)$} \\
\hline & \multicolumn{2}{|c|}{$0 \%$} & \multicolumn{2}{|c|}{$52 \%$} & \multirow{2}{*}{ Tolerant } & \multirow{2}{*}{ Sensitive } \\
\hline & Tolerant & Sensitive & Tolerant & Sensitive & & \\
\hline Leaf number/plant, 4 WAP & $14 \mathrm{a}$ & $15 \mathrm{a}$ & $12 d$ & $13 d$ & -14.3 & -13.3 \\
\hline Leaf number/plant, 6 WAP & $26 \mathrm{~b}$ & $30 \mathrm{a}$ & $18 d$ & $18 d$ & -30.8 & -40.0 \\
\hline Leaf number/plant, 8 WAP & $25 \mathrm{a}$ & $25 \mathrm{a}$ & $17 d$ & $16 d$ & -32.0 & -36.0 \\
\hline Leaf area $\left(\mathrm{cm}^{2} /\right.$ plant $), 4$ WAP & $313.8 \mathrm{a}$ & $329.8 \mathrm{a}$ & $271.7 d$ & $238.4 e$ & -13.4 & -27.7 \\
\hline Leaf area $\left(\mathrm{cm}^{2} /\right.$ plant $), 4$ WAP & $918.1 \mathrm{~b}$ & $1028.9 \mathrm{a}$ & $724.3 d$ & $703.7 e$ & -21.1 & -31.6 \\
\hline Leaf area $\left(\mathrm{cm}^{2} /\right.$ plant $), 4$ WAP & $996.2 \mathrm{a}$ & $840.4 b$ & $758.4 d$ & $614.4 d$ & -23.9 & -26.9 \\
\hline SLA $\left(\mathrm{cm}^{2} / \mathrm{g}\right), 4$ WAP & $275.9 a$ & $276.9 \mathrm{a}$ & $370.2 e$ & $396.7 d$ & 34.2 & 43.3 \\
\hline SLA $\left(\mathrm{cm}^{2} / \mathrm{g}\right), 6$ WAP & $211.4 \mathrm{a}$ & $219.1 \mathrm{a}$ & $299.5 d$ & $299.7 d$ & 41.6 & 36.8 \\
\hline SLA $\left(\mathrm{cm}^{2} / \mathrm{g}\right), 8$ WAP & $174.5 \mathrm{a}$ & $180.2 \mathrm{a}$ & $247.2 d$ & $239.2 d$ & 41.7 & 32.7 \\
\hline
\end{tabular}

in the same line, the number was followed the different letter was significant based on the $\mathrm{t}$ test $5 \%$. 
by MLG 429 and the lowest was performed by MLG 428 genotype, whereas the highest reducing in eight WAP was reached by MLG 310 genotype and the lowest was reached by VC 2768B genotype (Figure 3b,c).

Photosynthetic rate of the crops was different (Figure 4), at four WAP, photosynthetic rate of MLG 237 and MLG 429 genotypes was increased under the shading treatment, but
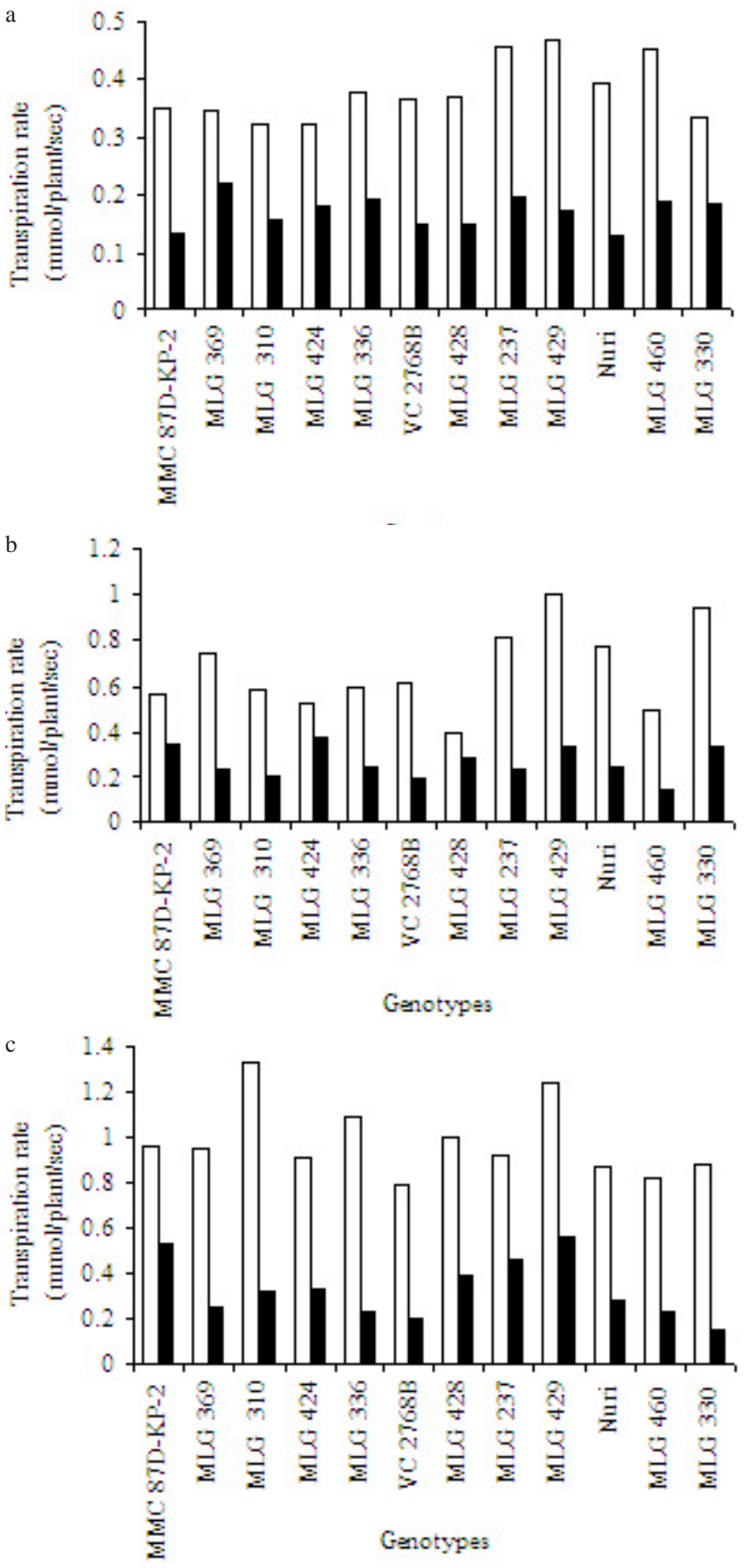

Figure 3. The transpiration rate (mmol/plant/sec) at: (a) 4 , (b) 6 , and (c) 8 WAP of twelve mungbean genotypes in without shading and 52\% shading level. $\square$ : without shading, $\mathbf{0}$ : $52 \%$ shading. the other genotypes were reduced (Figure 4a). At six and eight WAP, the highest reduction of photosynthetic rate was performed by MLG 429 and MLG 310 genotypes, and the lowest was found on MLG 428 and VC2768B genotypes (Figure $4 b, c)$.
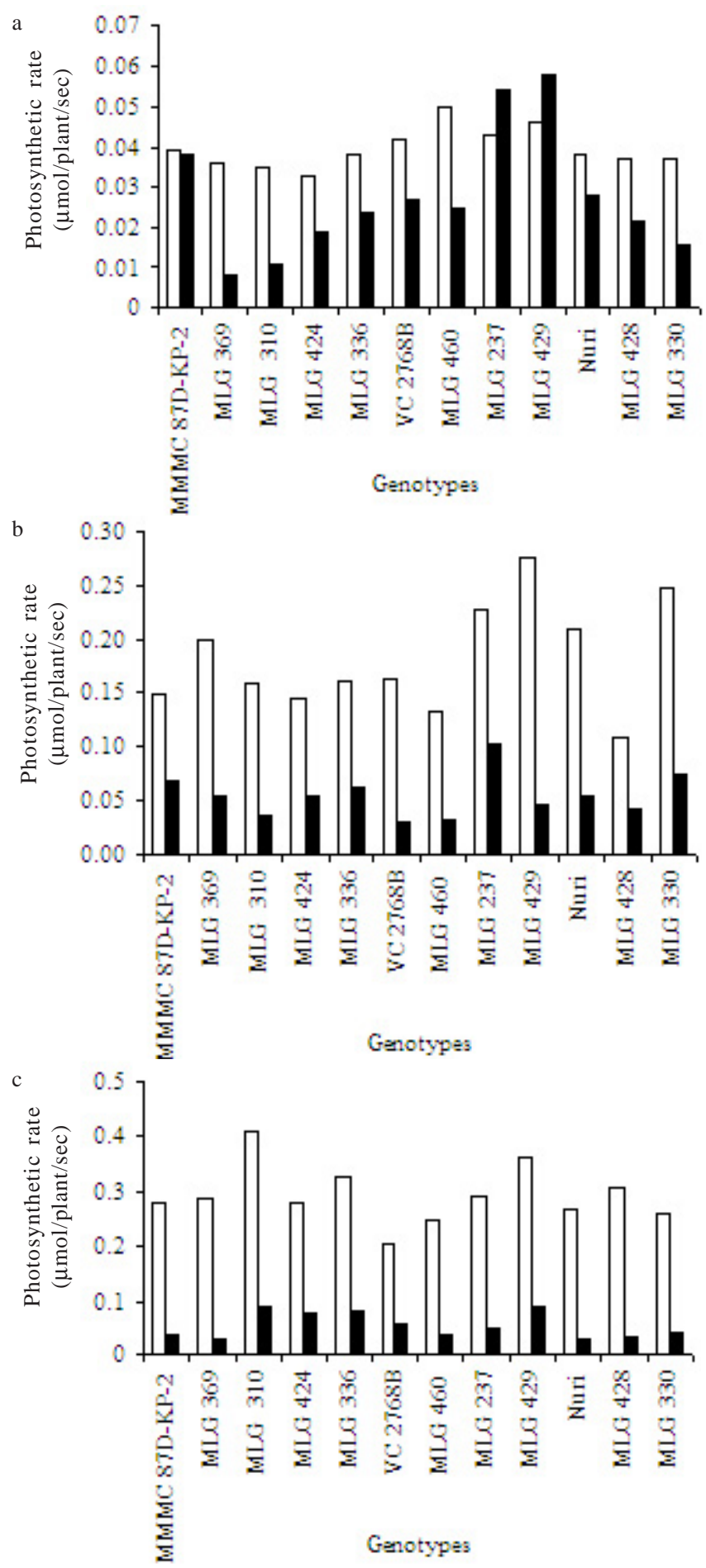

Figure 4 . The photosynthesis rate $(\mu \mathrm{mol} / \mathrm{plant} / \mathrm{sec}$ ) at: (a) 4, (b) 6 , and (c) 8 WAP of twelve mungbean genotypes in without shading and 52\% shading level. $\square$ : without shading, : $52 \%$ shading. 
Stomatal $\mathrm{CO}_{2}$ conductance rate of MLG 369, MLG 310 and MLG 336 genotypes was increased at four WAP. At six WAP, the conductance rate of $\mathrm{CO}_{2}$ stomata of all genotypes were descended, except for the MLG 424 genotype (Table 3). The highest reduction on conductance rate of $\mathrm{CO}_{2}$ stomata was reached by the MLG 429 genotype.

Chlorophyll-a content of each genotype was different (Figure 5) caused by different response of the genotypes to
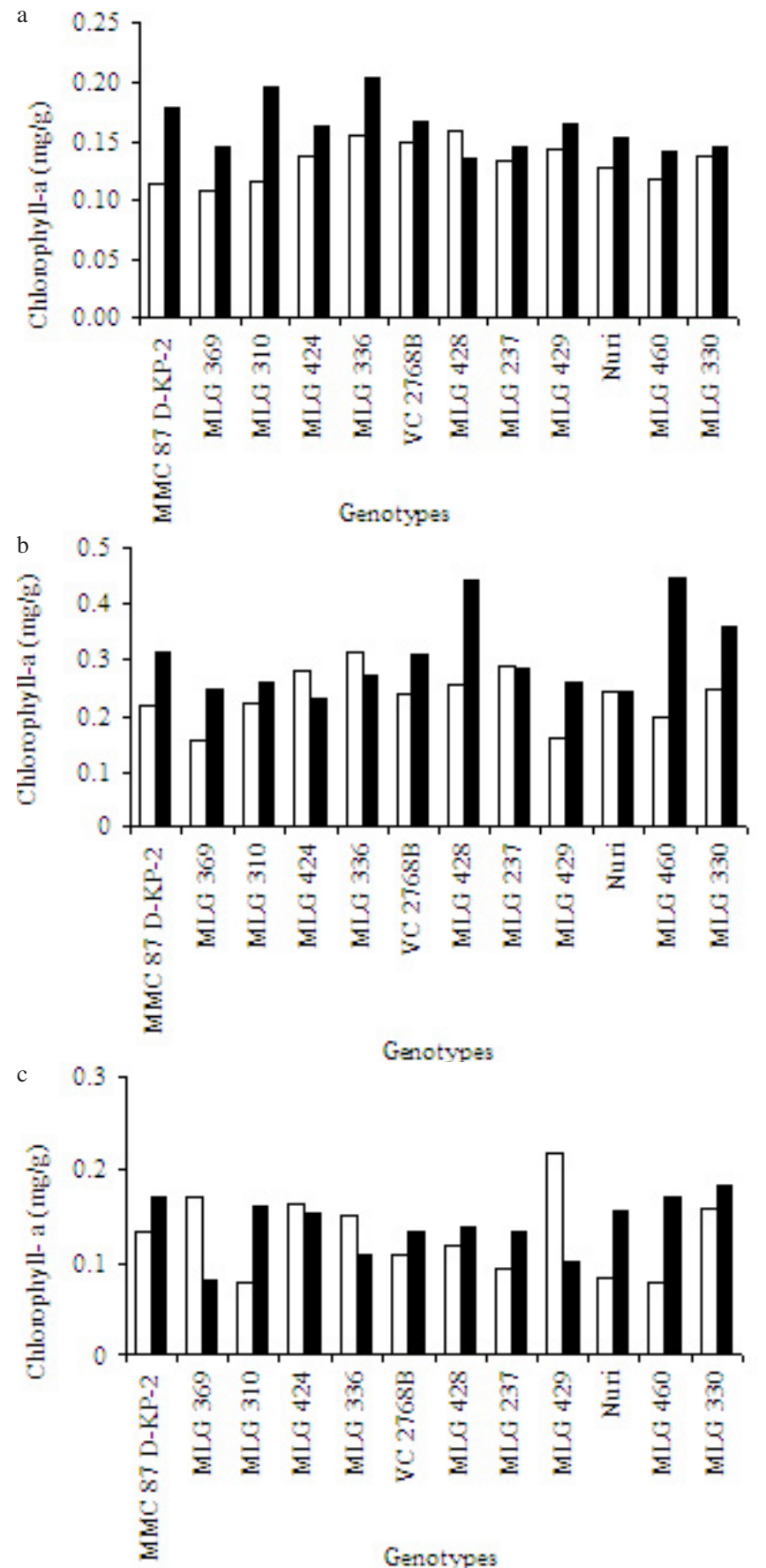

Figure 5. The chlorophyll a content (mg/g leave dry weight) at: (a) 4 , (b) 6 and (c) 8 WAP of twelve mungbean genotypes in without shading and 52\% shading level. $\square$ : without shading, a: $52 \%$ shading. shading indicated by different concentration of chlorophyll-a. The content of chlorophyll-a (Figure 5), chlorophyll-b (Figure 6) and the ratio of chlorophyll b/a (Table 4 ) was affected by the interaction between the genotype and shading.

PAR absorption rate of shading tolerant genotype was higher than that of sensitive genotype (Table 5). The PAR absorption rate of shading tolerant genotype was up to 61.73$74.87 \%$, whereas sensitive genotype was $64.37-78.32 \%$.
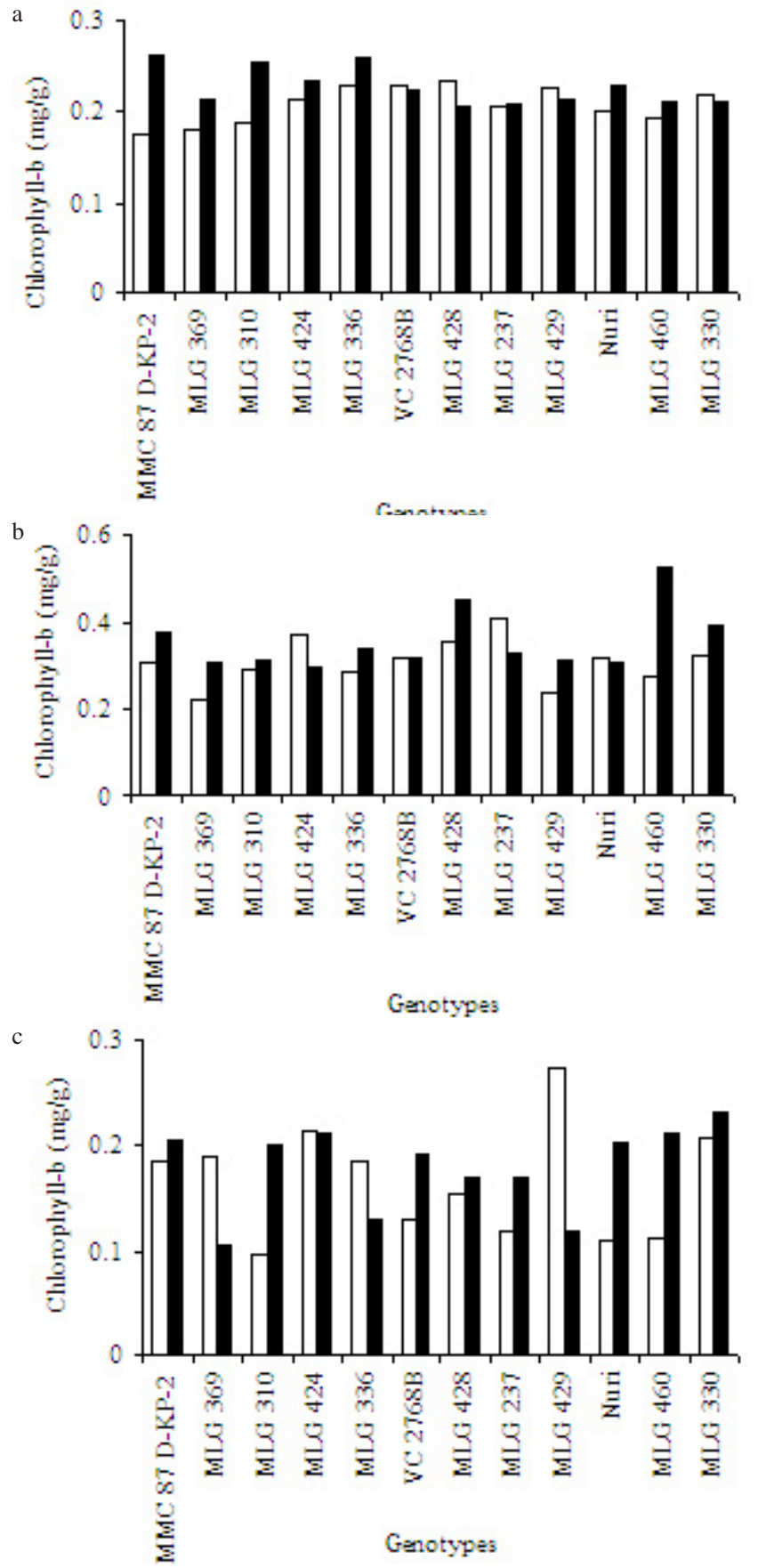

Figure 6 . The chlorophyll b content (mg/g leave dry weight) at: (a) 4 , (b) 6 , and (c) 8 WAP of twelve mungbean genotypes in without shading and $52 \%$ shading level. $\square$ : without shading, - $52 \%$ shading. 
The contrast analysis showed that the shading treatments caused reducing rate of PAR absorption, transpiration, photosynthesis, and $\mathrm{CO}_{2}$ stomata conductance (Table 5). The reduction of all parameters in tolerant genotype was smaller than that of sensitive genotype.

\section{DISCUSSION}

Leaves were crop photosynthetic apparatus harvesting light energy for the growth and photosyntate production through photosynthesis. Shading could cause reduction of

Table 3. The $\mathrm{CO}_{2}$ stomatal conductance (mol/plant/sec) of twelve mungbean genotypes at two shading levels

\begin{tabular}{|c|c|c|c|c|}
\hline \multirow{2}{*}{$\begin{array}{l}\text { Treatment } \\
\text { Shading levels (\%) }\end{array}$} & \multirow{2}{*}{ Genotypes } & \multicolumn{3}{|c|}{ Age (WAP) } \\
\hline & & 4 & 6 & 8 \\
\hline 0 & MMC 87 D-KP-2 & $0.033 \mathrm{abcd}$ & $0.071 \mathrm{~cd}$ & 0.028 bcde \\
\hline 0 & MLG 369 & $0.026 \mathrm{cdef}$ & $0.094 b$ & $0.026 \mathrm{cdef}$ \\
\hline 0 & MLG 310 & $0.025 \mathrm{cdef}$ & $0.074 \mathrm{~cd}$ & $0.035 \mathrm{abc}$ \\
\hline 0 & MLG 424 & $0.024 \mathrm{cdefgh}$ & $0.067 \mathrm{~cd}$ & $0.024 \mathrm{defg}$ \\
\hline 0 & MLG 336 & 0.021 defghi & $0.076 \mathrm{c}$ & $0.032 \mathrm{abcd}$ \\
\hline 0 & VC $2768 B$ & 0.028 bcde & $0.078 \mathrm{c}$ & $0.021 \mathrm{efg}$ \\
\hline 0 & MLG 428 & $0.040 \mathrm{ab}$ & $0.050 \mathrm{e}$ & $0.027 \mathrm{bcdef}$ \\
\hline 0 & MLG 237 & $0.029 \mathrm{bcde}$ & $0.103 b$ & $0.023 \mathrm{defg}$ \\
\hline 0 & MLG 429 & 0.021 defghi & $0.127 \mathrm{a}$ & $0.036 \mathrm{ab}$ \\
\hline 0 & Nuri* & 0.019efghij & $0.098 b$ & $0.023 \mathrm{defg}$ \\
\hline 0 & MLG 460* & $0.034 \mathrm{abc}$ & $0.063 \mathrm{~d}$ & $0.022 \mathrm{efg}$ \\
\hline 0 & MLG $330 *$ & 0.019efghij & $0.119 \mathrm{a}$ & $0.026 \mathrm{cdef}$ \\
\hline 52 & MMC 87 D-KP-2 & $0.009 \mathrm{ij}$ & $0.047 \mathrm{e}$ & $0.030 \mathrm{bcde}$ \\
\hline 52 & MLG 369 & $0.043 \mathrm{a}$ & $0.016 \mathrm{~h}$ & $0.011 \mathrm{hi}$ \\
\hline 52 & MLG 310 & $0.031 \mathrm{abcde}$ & $0.031 \mathrm{fg}$ & $0.022 \mathrm{efg}$ \\
\hline 52 & MLG 424 & 0.015 fghij & $0.073 \mathrm{~cd}$ & $0.018 \mathrm{fgh}$ \\
\hline 52 & MLG 336 & $0.026 \mathrm{cdef}$ & $0.022 \mathrm{gh}$ & $0.011 \mathrm{hi}$ \\
\hline 52 & VC 2768B & $0.007 \mathrm{j}$ & $0.035 \mathrm{f}$ & $0.007 \mathrm{i}$ \\
\hline 52 & MLG 428 & 0.013 ghij & $0.050 \mathrm{e}$ & $0.024 \mathrm{defg}$ \\
\hline 52 & MLG 237 & 0.015 fghij & $0.020 \mathrm{~h}$ & $0.028 \mathrm{bcde}$ \\
\hline 52 & MLG 429 & 0.014 fghij & $0.039 \mathrm{ef}$ & $0.039 \mathrm{a}$ \\
\hline 52 & Nuri* & $0.011 \mathrm{hij}$ & $0.032 \mathrm{fg}$ & $0.016 \mathrm{gh}$ \\
\hline 52 & MLG 460* & $0.012 \mathrm{hij}$ & $0.014 \mathrm{~h}$ & $0.011 \mathrm{hi}$ \\
\hline 52 & MLG $330^{*}$ & 0.014 fghij & $0.047 \mathrm{e}$ & $0.005 \mathrm{i}$ \\
\hline Variance coefficien & & 32.330 & 12.150 & 20.830 \\
\hline
\end{tabular}

In the same column, the number was followed the different letter showed was significantly according to the DMRT test 5\%; WAP: the week after planted; *: the genotype was sensitive to the shading.

Tablel 4. The chlorophyll b/a ratio of twelve mungbean genotypes at two shading levels

\begin{tabular}{|c|c|c|c|c|}
\hline \multirow{2}{*}{$\begin{array}{l}\text { Treatment } \\
\text { Shading levels (\%) }\end{array}$} & \multirow{2}{*}{ Genotypes } & \multicolumn{3}{|c|}{ Age (WAP) } \\
\hline & & 4 & 6 & 8 \\
\hline 0 & MMC 87 D-KP-2 & $1.542 \mathrm{fg}$ & $1.406 \mathrm{c}$ & $1.405 \mathrm{~b}$ \\
\hline 0 & MLG 369 & $1.649 \mathrm{a}$ & $1.439 b$ & $1.118 \mathrm{n}$ \\
\hline 0 & MLG 310 & $1.612 \mathrm{c}$ & $1.296 \mathrm{ef}$ & $1.230 \mathrm{ij}$ \\
\hline 0 & MLG 424 & $1.552 \mathrm{f}$ & $1.330 \mathrm{~d}$ & $1.301 \mathrm{ef}$ \\
\hline 0 & MLG 336 & $1.474 \mathrm{i}$ & $0.912 \mathrm{n}$ & $1.223 \mathrm{jk}$ \\
\hline 0 & VC 2768B & $1.524 \mathrm{~g}$ & $1.333 \mathrm{~d}$ & $1.186 \mathrm{~m}$ \\
\hline 0 & MLG 428 & $1.476 \mathrm{i}$ & $1.407 \mathrm{c}$ & $1.314 \mathrm{~d}$ \\
\hline 0 & MLG 237 & $1.539 \mathrm{fg}$ & $1.404 \mathrm{c}$ & $1.271 \mathrm{~g}$ \\
\hline 0 & MLG 429 & $1.571 \mathrm{e}$ & $1.485 \mathrm{a}$ & $1.256 \mathrm{~h}$ \\
\hline 0 & Nuri* & $1.574 \mathrm{de}$ & $1.305 \mathrm{e}$ & $1.294 \mathrm{f}$ \\
\hline 0 & MLG $460 *$ & $1.631 \mathrm{~b}$ & $1.397 \mathrm{c}$ & $1.429 \mathrm{a}$ \\
\hline 0 & MLG 330* & $1.591 \mathrm{~d}$ & $1.307 \mathrm{e}$ & $1.306 \mathrm{e}$ \\
\hline 52 & MMC 87 D-KP-2 & $1.466 \mathrm{i}$ & $1.205 \mathrm{i}$ & 1.2091 \\
\hline 52 & MLG 369 & $1.464 \mathrm{i}$ & $1.248 \mathrm{~h}$ & $1.314 \mathrm{~d}$ \\
\hline 52 & MLG 310 & 1.3011 & $1.194 \mathrm{i}$ & $1.253 \mathrm{~h}$ \\
\hline 52 & MLG 424 & $1.430 \mathrm{j}$ & $1.287 \mathrm{f}$ & $1.376 \mathrm{c}$ \\
\hline 52 & MLG 336 & $1.277 \mathrm{~m}$ & $1.243 \mathrm{~h}$ & 1.2011 \\
\hline 52 & VC $2768 B$ & $1.352 \mathrm{k}$ & $1.033 \mathrm{~m}$ & $1.423 \mathrm{a}$ \\
\hline 52 & MLG 428 & $1.529 \mathrm{~g}$ & $1.017 \mathrm{~m}$ & $1.219 \mathrm{k}$ \\
\hline 52 & MLG 237 & $1.430 \mathrm{j}$ & $1.161 \mathrm{k}$ & $1.260 \mathrm{~h}$ \\
\hline 52 & MLG 429 & 1.3101 & $1.198 \mathrm{i}$ & $1.191 \mathrm{~m}$ \\
\hline 52 & Nuri* & $1.499 \mathrm{~h}$ & $1.266 \mathrm{~g}$ & $1.304 \mathrm{e}$ \\
\hline 52 & MLG 460* & $1.501 \mathrm{~h}$ & $1.178 \mathrm{j}$ & $1.235 \mathrm{i}$ \\
\hline 52 & MLG $330 *$ & $1.442 \mathrm{j}$ & 1.0881 & $1.271 \mathrm{~g}$ \\
\hline Variance coefficien & & 0.680 & 0.720 & 0.400 \\
\hline
\end{tabular}

In the same column, the number was followed the different letter showed was significantly according to the DMRT test 5\%; WAP: the week after planted; *: the genotype was sensitive to the shading. 
Table 5. The contrast test of the physiological characters of inter group mungbean genotypes in the 0 and $52 \%$ shading lavels

\begin{tabular}{|c|c|c|c|c|c|c|}
\hline \multirow{3}{*}{ Quantitative characters } & \multicolumn{4}{|c|}{ Shading levels } & \multicolumn{2}{|c|}{ Percentage change $(\%)$} \\
\hline & \multicolumn{2}{|c|}{$0 \%$} & \multicolumn{2}{|c|}{$52 \%$} & Tolerant & Sensitive \\
\hline & Tolerant & Sensitive & Tolerant & Sensitive & Toterait & semsitive \\
\hline PAR rate $(\mu \mathrm{mol} / \mathrm{plant} / \mathrm{sec}), 4 \mathrm{WAP}$ & $63.83 \mathrm{a}$ & $67.06 \mathrm{a}$ & $16.04 d$ & $14.54 e$ & $-74.87 p$ & $-78.32 \mathrm{p}$ \\
\hline PAR rate $(\mu \mathrm{mol} / \mathrm{plant} / \mathrm{sec}), 6 \mathrm{WAP}$ & $50.20 \mathrm{~b}$ & $54.89 a$ & $19.21 d$ & $19.56 d$ & $-61.73 p$ & $-64.37 p$ \\
\hline PAR rate $(\mu \mathrm{mol} / \mathrm{plant} / \mathrm{sec}), 8 \mathrm{WAP}$ & $129.85 \mathrm{a}$ & $109.68 \mathrm{~b}$ & $45.13 d$ & $36.10 e$ & $-65.24 q$ & $-67.09 p$ \\
\hline Transpiration rate $(\mathrm{mmol} / \mathrm{plant} / \mathrm{sec}), 4 \mathrm{WAP}$ & $0.38 \mathrm{a}$ & $0.39 \mathrm{a}$ & $0.17 d$ & $0.17 d$ & $-55.26 \mathrm{p}$ & $-56.41 \mathrm{p}$ \\
\hline Transpiration rate $(\mathrm{mmol} / \mathrm{plant} / \mathrm{sec}), 6$ WAP & $0.65 b$ & $0.74 \mathrm{a}$ & $0.27 d$ & $0.24 d$ & $-58.46 q$ & $-67.57 p$ \\
\hline Transpiration rate $(\mathrm{mmol} / \mathrm{plant} / \mathrm{sec}), 8 \mathrm{WAP}$ & $1.02 \mathrm{a}$ & $0.86 \mathrm{a}$ & $0.36 d$ & $0.22 e$ & $-64.71 p$ & $-74.42 \mathrm{p}$ \\
\hline Photosyntesis rate $(\mu \mathrm{mol} / \mathrm{plant} / \mathrm{sec}), 4$ WAP & $0.039 \mathrm{a}$ & $0.042 \mathrm{a}$ & $0.029 d$ & $0.023 e$ & $-25.64 q$ & $-45.24 p$ \\
\hline Photosyntesis rate $(\mu \mathrm{mol} / \mathrm{plant} / \mathrm{sec}), 6$ WAP & $0.177 b$ & $0.197 \mathrm{a}$ & $0.055 d$ & $0.054 d$ & $-68.93 q$ & $-72.59 p$ \\
\hline Photosyntesis rate $(\mu \mathrm{mol} / \mathrm{plant} / \mathrm{sec}), 8$ WAP & $0.306 \mathrm{a}$ & $0.260 \mathrm{~b}$ & $0.061 d$ & $0.034 e$ & $-80.07 \mathrm{p}$ & $-86.92 p$ \\
\hline Currant $\mathrm{CO}_{2}$ stomata $(\mathrm{mol} / \mathrm{plant} / \mathrm{sec}), 4$ WAP & $0.028 \mathrm{a}$ & $0.024 \mathrm{a}$ & $0.019 \mathrm{~d}$ & $0.012 \mathrm{e}$ & $-32.14 p$ & $-50.00 p$ \\
\hline Currant $\mathrm{CO}_{2}$ stomata $(\mathrm{mol} / \mathrm{plant} / \mathrm{sec}), 6$ WAP & $0.082 b$ & $0.093 \mathrm{a}$ & $0.037 \mathrm{~d}$ & $0.031 \mathrm{~d}$ & $-54.88 \mathrm{q}$ & $-66.67 p$ \\
\hline Currant $\mathrm{CO}_{2}$ stomata $(\mathrm{mol} / \mathrm{plant} / \mathrm{sec}), 8$ WAP & $0.028 \mathrm{a}$ & $0.024 b$ & $0.021 \mathrm{~d}$ & $0.011 \mathrm{e}$ & $-25.00 p$ & $-54.17 p$ \\
\hline Chlorophyll a (mg/g leaf dry weight), 4 WAP & $0.14 \mathrm{a}$ & $0.13 \mathrm{a}$ & $0.170 \mathrm{~d}$ & $0.15 \mathrm{e}$ & 21.43 & 15.38 \\
\hline Chlorophyll a (mg/g leaf dry weight), 6 WAP & $0.24 \mathrm{a}$ & $0.23 \mathrm{a}$ & $0.29 \mathrm{e}$ & $0.35 \mathrm{~d}$ & 20.83 & 52.17 \\
\hline Chlorophyll a (mg/g leaf dry weight), 8 WAP & $0.14 \mathrm{a}$ & $0.11 b$ & $0.13 \mathrm{~d}$ & $0.17 \mathrm{~d}$ & -7.14 & 54.54 \\
\hline Chlorophyll b (mg/g leaf dry weight), 4 WAP & $0.21 \mathrm{a}$ & $0.20 \mathrm{a}$ & $0.23 \mathrm{~d}$ & $0.22 \mathrm{e}$ & 9.52 & 10.00 \\
\hline Chlorophyll b (mg/g leaf dry weight), 6 WAP & $0.31 \mathrm{a}$ & $0.31 \mathrm{a}$ & $0.34 \mathrm{e}$ & $0.41 \mathrm{~d}$ & 9.68 & 32.26 \\
\hline Chlorophyll b (mg/g leaf dry weight), 8 WAP & $0.17 \mathrm{a}$ & $0.14 b$ & $0.17 \mathrm{e}$ & $0.22 \mathrm{~d}$ & 0 & 57.14 \\
\hline Chlorophyll b/a ratio, 4 WAP & $1.540 \mathrm{~b}$ & $1.599 \mathrm{a}$ & $1.396 \mathrm{e}$ & $1.479 \mathrm{~d}$ & $-9.35 p$ & $-7.50 p$ \\
\hline Chlorophyll b/a ratio, 6 WAP & $1.335 \mathrm{a}$ & $1.336 \mathrm{a}$ & $1.179 \mathrm{~d}$ & $1.177 \mathrm{~d}$ & $-11.91 \mathrm{p}$ & $-11.90 p$ \\
\hline
\end{tabular}

The number was followed with the same letter to the same line was not significant according to 5\% test, WAP: week after planted, PAR: photosynthetically active radiation, the sign of the negative (-) showed the reduction.

leaf number and area. Leaf area reduction had direct impact on reduction photosyntate production. Several researches showed that increasing of leaves area will increase the rate of net photosynthesis (Widiastuti et al. 2004; Sulandjari et al. 2005).

The different genotypes will response differently to light stress. The shading tolerant mungbean genotypes had good response to light stress so that the growth and development of the leaves were better than that of sensitive genotypes. The shading tolerant mungbean genotypes had bigger and thicker leaves than that of sensitive genotypes. Souza and Valio (2003) also reported that leaf thickness of shading adapted plant could live in light stress condition and the thickness of their leaves was not change. The light stress could be avoided through increasing of interception light efficiency, total light interception by increasing of leaf area, proportion of leaf area per unit plant tissue, and percentage of the light absorbed for photosynthesis. Efficiency of light interception was reached by reducing of reflected and transmitted light through reduction of leaves trichoma number and increasing of chlorophyll content.

Reducing of quantity and the quality of the sunlight will influence crop physiological process on opening and closing of stomata, rate of transpiration, photosynthetic dynamics, and stomatal conductance (Rajapakse et al. 1999; Dong \& He 2003). Stomatal movements to enable gas exchange with the atmosphere are mainly controlled by light. The exchange of water vapour, $\mathrm{CO}_{2}$, and $\mathrm{O}_{2}$ is limited to stomatal pores, which are minute intercellular openings bounded by two kidneyshaped specialized epidermis cells called the guard cells (Bolhar-Nordenkampf \& Draxler 1993). When the transpiration rate was higher, the stomata was closed. In this condition, $\mathrm{CO}_{2}$ that entered decreased, and the photosynthesis rate would be descend. Under conditions of high rates of transpiration, the leaves may temporarily wilt and close their stomata. At times entry of $\mathrm{CO}_{2}$ is reduced, and the rate of photosynthesis will drop.

The rate of the photosynthesis is influenced by light intensity, $\mathrm{CO}_{2}$ concentration and temperature. Photosynthesis does not occur in the absence of light, but as the intensity of irradiation will increase the rate of photosynthesis. The rate of the photosynthesis will reduce as well as the shading level is increased (Islam et al. 1999; de Alvarenga et al. 2003). Reducion of the sunlight will reduce the rate of photosynthesis. The sunllight was the main energy source in the photosynthesis process, so that the light intensity could control photosynthesis (Xu \& Shen 1999). Light radiation influences the growth and development of the crop through photosynthesis. Plants growing under shade exhibits lower biomass production compared with plants growing under higher irradiance (Huante \& Rincon 1998).

Radiant energy with wavelength of 400-700 $\mathrm{nm}$ plays an important role in the photosynthesis process. Therefore, rate of the PAR absorption was increased as well as the photosynthesis rate. Green plants capture solar energy and convert it into chemical energy by the photosynthesis process. During photosynthesis, $\mathrm{CO}_{2}$ and water are transformed into simple carbohydrates and oxygen gas.

The leaves of plants are photosynthetically active organ, which are able to store absorbed solar energy in reduced organic compounds (Bolhar-Nordenkampf \& Draxler 1993). The wider and flat leaf surface were enabled to maximize absorption of light by increasing of leaf area (Jones \& McLeod 1990).

All the photosynthetic cells contained one or more the chlorophyll. In the process of photosynthesis, chlorophyll 
played in the light energy absorption and change this energy into the chemical energy in the photosynthesis process (Bolhar-Nordenkampf \& Oquist 1993).

Chlorophyll is green pigments, which plays in the light energy absorption. There are several kinds of chlorophyll i.e. chlorophyll a, b, c, and d, however, the most abundant are chlorophyll-a $\left(\mathrm{C}_{55} \mathrm{H}_{72} \mathrm{O}_{5} \mathrm{~N}_{4} \mathrm{Mg}\right)$ and $-\mathrm{b}\left(\mathrm{C}_{55} \mathrm{H}_{70} \mathrm{O}_{6} \mathrm{~N}_{4} \mathrm{Mg}\right)$. Chlorophyll-a is dark green in color, whereas chlorophyll-b is light green. The shading could increase content of chlorophyll-a and -b per dry weight of leaves (Goncalves et al. 2001; Danesi et al. 2004). Increasing of chlorophyll content was a mechanism in order to increase the light interception to maintain normal life of the crop under shading condition. This increased content of the chlorophyll was to enhance efficiency of the PAR absorption indicating adaptation of crops to the low light intensity (Cartechini \& Palliotti 1995). Two mechanisms of plant to avoid low light intensity are by increasing the total light absorption for the photosynthesis process, and by reducing reflected and transmitted light.

\section{ACKNOWLEDGEMENT}

The Author thanks to Kuncoro Adie for help in collecting plant physiology data. This research was supported by the Indonesian Agency for Agricultural Research and Development (IAARD).

\section{REFFERENCES}

Bolhar-Nordenkampf HR, Draxler G. 1993. Functional leaf anatomy. In: Hall DO, Scurlock JMO, Bolhar-Nordenkampf HR, Leegood $\mathrm{RC}$, Long SP (eds). Photosynthesis and Production in a Changing Environment. A Field and Laboratory Manual. London: Chapman \& Hall. p 91-112.

Bolhar-Nordenkampf HR, Oquist G. 1993. Chlorophyll fluorescence as a tool in photosynthesis research. In: Hall DO, Scurlock JMO, Bolhar-Nordenkampf HR, Leegood RC, Long SP (eds). Photosynthesis and Production in a Changing Environment. A Field and Laboratory Manual. London: Chapman \& Hall. p 193-206.

Cartechini A, Palliotti A. 1995. Effect of shading on vine morphology and productivity and leaf gas exchange characteristics in grapevines in the field. Am J Enol Vitic 46:227-234.

Danesi EDG, Rangel-Yagui CO, Carvalho JCM, Sato S. 2004. Effect of reducing the light intensity on the growth and production of chlorophyll by Spirulina platensis. J Biomass Bioenergy 26:329335. de Alvarenga AA, de Castro EM, de Castro Lima Junior É, Magalhães MM. 2003. Effects of different light levels on the initial growth and photosynthesis of Croton urucurana Baill. in southeastern Brazil. Rev Árvore 27:53-57.

de Carvalhu Gonsales JF, de Sousa Barreto DC, dos Santos Junior, Vernandes AV, de Tarso Barbosa Sampaio P. 2005. Growth, photosynthesis and stress indicators in young rosewood plants $(A$. rosaeodora Ducke) under different light intensities. Baz J Plant Physiol 17:325-334.

Dong M, He WM. 2003. Physiological acclimation and growth response to partial shading in Salix matsudana in $\mathrm{Mu}$ us sandland in China Trees 17:87-93.

Gonçalves JFC, Marenco RA, Vieira G. 2001. Concentration of photosynthetic pigments and chlorophyll fluorescence of mahogany and tonka bean under two light environments. Braz J Plant Physiol 13:149-157.

Huante P, Rincón E. 1998. Responses to Light changes in tropical deciduous woody young plant with contrasting growth rate. Oecologia 113:53-66.

Islam MS, Hamid A, Hashem A, Ahmed ZU. 1999. Shading effect on photosynthetic rate and gas exchange characteristics of mungbean and blackgram. Annals Bangladesh Agriculture 9:25-34.

Jones RH, McLeod KW. 1990. Growth and photosynthetic responses to a range of light environments in Chinese tollow tree and Carolina ash seedlings. Forest Science 36:851-862.

Poorter L, Oberbauer SF, Clark DB. 1995. Leaf optical properties along a vertical gradient in a tropical rain forest canopy in Costa Rica. Am J Bot 82:1257-1263.

Rajapakse NC, Young RE, McMahon MJ, Oi R. 1999. Plant height control by photoselective filters: Current status and future prospects. Hort Technol 9:618-624.

Sitompul SM, Guritno B. 1991. Analisis Pertumbuhan Tanaman. Malang, Fakultas Pertanian Universitas Brawijaya.

Souza RP, Valio IFM. 2003. Leaf optical properties as affected by shade in saplings of six tropical tree species differing in successional status. Braz J Plant Physiol 15:49-54.

St-Jacques C, Labrecque M, Bellefleur P. 1991. Plasticity of leaf absorbance in some broadleaf tree seedlings. Bot Gaz 152:195 202.

Sulandjari, Pramono S, Wisnubroto S, Indradewa D. 2005. Hubungan mikroklimat dengan pertumbuhan dan hasil Pule Landak. $J$ Agrosains 7:71-76.

Thompson WA, huang LK, Kriedemann PE. 1992. Photosynthetic response to light and nutriens in sun tolerant and shade tolerant rainforest trees. Leaf gas exchange and component processes of photosynthesis. Aust J Plant Physiol 19:19-42.

Widiastuti L, Tohari, Sulistyaningsih E. 2004. Pengaruh intensitas cahaya dan kadar daminosida terhadap iklim mikro dan pertumbuhan tanaman Krisn dalam pot. J Ilmu Pertanian 11:3442.

Xu, Da-Quan, Yun-Kang Shen. 1999. Light stress: Photoinhibition of photosynthesis in plant under natural conditions. In: Pessarakli M (ed). Hand book of Plant and Crop Stress. 2nd. New York, Basel: Marcel Dekker, Inc. p 483-497. 